\title{
Simulação computacional de campos ultra-sônicos
}

(Computational simulation of ultrasonic fields)

\author{
Francisco J. Arnold 1 e Carlos A. Pelá ${ }^{2}$ \\ ${ }^{1}$ Centro Superior de Educação Tecnológica, UNICAMP, Limeira, SP, Brasil \\ ${ }^{2}$ Faculdade de Filosofia, Ciências e Letras de Ribeirão Preto, USP, Ribeirão Preto, SP, Brasil \\ Recebido em 23/12/03; Aceito em 01/06/04
}

\begin{abstract}
Quando um transdutor eletro-acústico é excitado eletricamente, ele produz no meio de propagação onde está inserido um campo acústico. Através do conhecimento dos campos acústicos pode-se colher importantes subsídios para projetos de transdutores e estudos da interação com os meios de propagação. Neste trabalho é apresentada uma proposta de atividade interdisciplinar envolvendo conteúdos de Física, Matemática e Computação ministrados nas disciplinas introdutórias dos cursos superiores de ciências exatas e tecnológicas. O trabalho propõe a discussão dos fenômenos de interferência ondulatória, o cálculo do campo acústico usando coordenadas cilíndricas e a programação do método matemático através de instruções básicas de uma linguagem de programação. As simulações de alguns campos acústicos são apresentadas na forma de gráficos bi e tridimensionais e os resultados são discutidos.
\end{abstract}

Palavras-chave: campo ultra-sônico, simulação computacional.

When an electro-acoustical transducer is electrically supplied, it yields an acoustic field in the propagation medium. The acoustic field knowledge allows us to obtain significant help to transducers design and propagation medium interaction studies. In this paper, it is shown a proposal of classroom work involving the studied contents of physics, mathematic and computing in the introductory lessons of degree course of exact science. It is proposed the discussion about wave interference phenomena, the determination of acoustic field using cylindrical coordinates and the software of the mathematical method applying basic instructions of a computational language. The simulations of some acoustic fields are shown in bi and tri-dimensional graphics and the results are discussed.

Keywords: ultra-sonic field, computational simulation.

\section{Introdução}

$\mathrm{O}$ ensino de disciplinas ligadas à matéria Física em semestres iniciais de cursos superiores passa, atualmente, por uma séria dificuldade ligada a deficiência com o trato matemático necessário para acompanhar, com a devida profundidade, o conteúdo a ser estudado nestas disciplinas [1]. A experiência docente tem demonstrado que isto traz graves conseqüências, pois dificulta o entendimento da complementação entre Física e Matemática.

O interesse pela aplicação e a necessidade de busca e de transformação do processo de ensino e aprendizagem tem levado professores e pesquisadores, das diversas áreas do conhecimento, a abordarem situações interdisciplinares como estratégias de ensino, propondo currículos com enfoques diferentes e, sobretudo, adotando uma abordagem ligada às atividades práticas e, muitas vezes, de cunho tecnológico, vinculadas às demais áreas do conhecimento.

O elo entre os problemas da Física e da Matemática constitui um importante fator motivador para o aprendizado de ambas as disciplinas e revela, principalmente, o aspecto prático de conceitos matemáticos que são ensinados, por muitas vezes, restritos a situações impalpáveis. A modelagem de um problema físico, como o dos campos acústicos, em conjunto com a sua simulação computacional, constitui-se numa atividade interdisciplinar que reúne os conhecimentos dessas matérias. Essas simulações também encontram uma

\footnotetext{
${ }^{1}$ Enviar correspondência para Francisco J. Arnold. E-mail: arnold@ ceset.unicamp.br.
} 
extensa gama de aplicações tecnológicas, entre elas pode-se destacar o ultra-som.

Os campos acústicos são determinados através da integral de Rayleigh [2]. As simulações computacionais dos campos acústicos são peças fundamentais para o entendimento de sistemas acústicos como um todo e o problema em si desperta grande interesse matemático. Existem vários métodos matemáticos para a determinação dos campos acústicos [3], todavia, os mais populares, empregam a integração direta e a convolução.

A integral de Rayleigh é calculada sobre a superfície da fonte acústica. A aplicação desta metodologia vincula-se ao princípio de Huygens [4] e consiste em dividir a fonte acústica em elementos de superfície e em somar as contribuições de cada um deles em um certo ponto do meio de propagação [5]. Por se tratar de uma integral dupla, o trabalho computacional para calculá-la envolve grandes quantidades de memória, principalmente quando os comprimentos de onda são pequenos em relação às dimensões da fonte, o que demanda uma malha com muitos pontos para se obter precisão. A literatura técnica tem depositado nas últimas décadas grandes esforços no sentido de melhorar a eficiência do trabalho computacional. Estes esforços estão direcionados a simplificações algébricas na modelagem de natureza numérica e analítica, e ao desenvolvimento de algoritmos apropriados [6-13].

A outra metodologia é baseada no cálculo de uma convolução através do produto de transformadas de Fourier [14-17]. Neste método, a distribuição de pressão sobre uma superfície plana é calculada a partir do produto de um espectro de ondas de outro plano paralelo multiplicado por um fator de fase. Esta técnica apresenta alta eficiência computacional devido aos algoritmos de FFT (Fast Fourier Transform). Todavia, devido aos possíveis erros decorrentes do uso de transformadas de Fourier discretas, cuidados especiais devem ser tomados para se obter resultados precisos [1820].

A distribuição dos níveis de pressão em um campo acústico é embasada pelos princípios da interferência das ondas e seu cálculo, no caso de ser produzida por uma fonte circular, apresenta particularidades que favorecem o uso de coordenadas cilíndricas. A determinação do nível de pressão em um dado ponto do meio de propagação é feita através da somatória fasorial das contribuições de fontes infinitesimais que compõem a superfície do transdutor, tratandose, portanto, de uma aplicação com a metodologia da integração direta. Isto nos faz reportar a diversos tópicos estudados nas disciplinas introdutórias dos cur- sos da área de ciências exatas: ondas mecânicas, coordenadas cilíndricas e integração numérica. Agregase a isso a necessidade de resolver o problema por meios computacionais em virtude da grande quantidade de cálculos e, portanto, instruções elementares da programação de computadores podem ser inseridas em um algoritmo para simular uma situação física. Os resultados são apresentados em gráficos bi e tridimensionais. O uso de gráficos tridimensionais permite ao aluno um primeiro contato com a interpretação desse tipo de representação gráfica.

A presente proposta apresenta a modelagem e a simulação computacional de campos acústicos produzidos por fontes acústicas planas com vibração superficial uniforme. Esta proposta reúne vários tópicos ministrados em disciplinas introdutórias das matérias Física, Matemática e Computação de cursos das áreas de ciências exatas e tecnológicas, o que visa estimular a realização de um trabalho interdisciplinar.

\section{Teoria}

Um transdutor eletro-acústico é um dispositivo que produz ondas acústicas quando excitado por sinais elétricos apropriados. Existem vários tipos de transdutores eletro-acústicos, contudo, neste trabalho são destacados aqueles empregados na concepção de geradores de ultra-som. O ultra-som (ondas sonoras com freqüências superiores a $20 \mathrm{kHz}$ ) é aplicado em várias áreas tecnológicas como a médica, a industrial e a militar [21]. Em grande parte dessas aplicações, esses transdutores são compostos por cerâmicas piezoelétricas na forma de disco. A Fig. 1 apresenta amostras comerciais destes discos, com diâmetros e espessuras diferentes, nas quais pode-se notar os eletrodos metálicos depositados nas suas faces planas. Estes discos normalmente encontram-se encerrados por invólucros metálicos e associados a outras peças não piezoelétricas como retaguardas e concentradores.

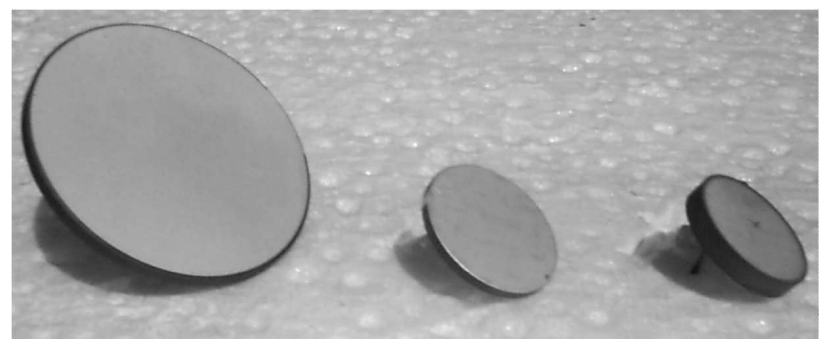

Figura 1 - Fotografia de discos cerâmicos piezoelétricos. Na imagem pode-se reparar a diferença entre os diâmetros e espessuras das amostras, o que lhes confere diferentes freqüências de vibração. 
Estando o transdutor inserido em um meio de propagação de ondas e aplicando-se aos eletrodos, do disco cerâmico, uma excitação elétrica senoidal na freqüência fundamental do modo de vibração espessura do elemento piezoelétrico, a superfície plana do elemento vibra, impondo, a cada ponto pertencente ao meio de propagação das ondas, níveis de pressão e velocidade de vibração de partículas. Diz-se, então, que foi estabelecido um campo acústico.

A superfície plana de um transdutor piezelétrico pode ser considerada como um conjunto de fontes pontuais esféricas que geram ondas descritas pela Eq. (1)

$$
p(R, t)=\frac{A}{R} e^{i(\omega t-k R)}
$$

onde $p(R, t)$ é a pressão em um ponto do meio de propagação localizado a uma distância $R$ da fonte acústica no tempo $t ; R$ é a distância entre a fonte acústica e o ponto do meio de propagação; $A$ é uma constante; $i$ é o número complexo; $\omega$ é a freqüência angular dada por $\omega=2 \pi f$, onde $f$ é a freqüência de vibração da fonte; $k$ é o número de onda definido como $k=2 \pi f / c$, onde $c$ é a velocidade de propagação das ondas no meio e $t$ é o tempo.

Para se determinar a pressão resultante em um ponto $\mathrm{P}\left(x_{P}, y_{P}, z_{P}\right)$, pertencente a um meio de propagação homogêneo e isotrópico, é necessário proceder a soma em amplitude e fase das contribuições individuais de cada uma das fontes virtuais pontuais. Na Fig. 2 são mostrados raios ligando algumas fontes pontuais presentes na superfície da fonte acústica, posicionada no plano xy, ao ponto P. O comprimento de cada raio define a amplitude e a fase de cada contribuição ao ponto $\mathrm{P}$. O conjunto dos níveis de pressão em todos os pontos do meio de propagação constitui o campo acústico gerado pelo transdutor.

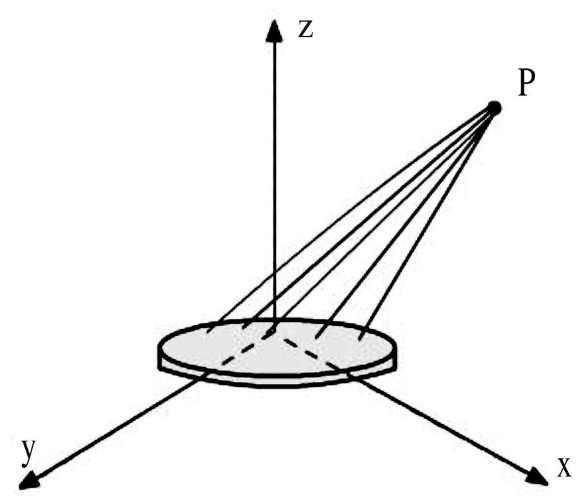

Figura 2 - Raios ligando fontes pontuais da superfície da fonte acústica ao ponto $\mathrm{P}$ pertencente ao meio de propagação.

A determinação do nível de pressão pode ser simplificada observando-se que as fontes pontuais eqüidis- tantes a $\mathrm{P}$ contribuem com amplitudes e fases iguais. Sendo assim, imaginando-se que em $\mathrm{P}$ esteja centrada uma esfera virtual de raio variável $R$, encontrarse-á um conjunto de fontes com as mesmas características de amplitude e fase como resultado da intersecção dessa esfera com a superfície do transdutor. Isto é, para cada ponto $\mathrm{P}$ deve-se fazer uma somatória das contribuições dos elementos resultantes dessa intersecção, levando-se em conta a velocidade de vibração das fontes pontuais e da distância entre estas e P [6,22]. Assim pode-se escrever a pressão $p$ como:

$$
p\left(x_{p}, y_{p}, z_{p}\right)=\psi \sum_{i} v_{i} \frac{L_{i}}{R_{i}}
$$

onde $\psi$ é uma constante de proporcionalidade; $v_{i}$ é a velocidade de vibração das fontes dada na forma fasorial por $v_{i}=V e^{i 2 \pi f R_{i} / c} ; L_{i}$ é o comprimento do arco resultante da i-ésima intersecção obtida; $R_{i}$ é a distância entre o arco obtido da intersecção e o ponto $\mathrm{P}$ e $V$ é amplitude de vibração das fontes.

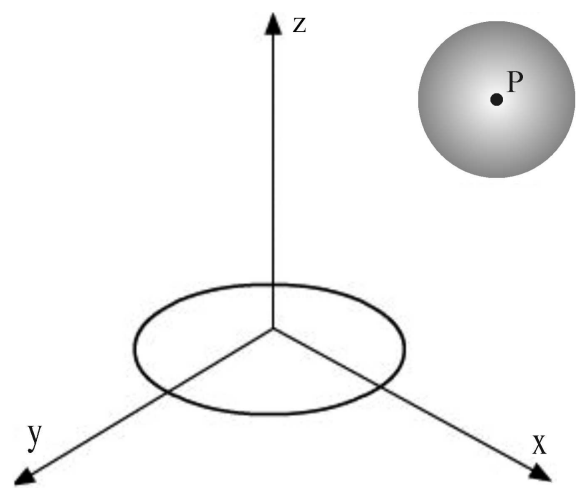

Figura 3 - Transdutor posicionado no sistema de coordenadas e esfera virtual centrada em um ponto do meio de propagação $P$.

Para efeito de cálculo pode-se considerar um sistema de coordenadas retangulares, onde o transdutor circular esteja posicionado no plano xy em $z=0$, centrado em $(0,0)$, como mostra a Fig. 3. Quando o raio da esfera for maior que $z_{p}$, a esfera intersecciona o plano xy. Sob certas condições, esta intersecção produz arcos ou circunferências internos à circunferência que define o transdutor, cujos pontos apresentam $z=0$ em comum.

A Fig. 4 ilustra os dois casos que podem ocorrer em termos de intersecção relacionados à posição do centro da esfera. Sendo $\mathrm{P}^{\prime}=\left(x_{p}, y_{p}, 0\right)$ o ponto $\mathrm{P}$ projetado no plano xy, no caso (a), onde P' está localizado externo à superfície do transdutor, só ocorrerá intersecção quando o raio da circunferência projetada no plano xy estiver com comprimento entre P'M e P'N. No caso (b), P' encontra-se no interior do transdutor, 
assim, se o raio da circunferência projetada estiver entre 0 e P'M, as fontes que produzirão contribuições para a pressão em $\mathrm{P}$ estarão compondo um circunferência interna ao transdutor. A intersecção entre as circunferências do transdutor e a projetada ocorrerá quando o raio da segunda estiver entre P'M e P'N.
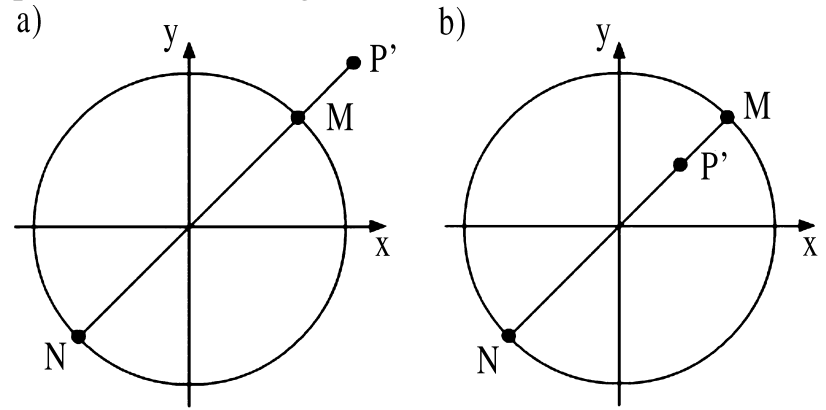

Figura 4 - Casos de projeção do ponto $\mathrm{P}$ sobre o plano xy a considerar: a) $\mathrm{P}^{\prime}$ está externo ao transdutor; b) $\mathrm{P}^{\prime}$ está interno ao transdutor.

A geometria circular envolvida neste problema sugere o uso de coordenadas cilíndricas [23]. Cada ponto do meio de propagação descrito por coordenadas cilíndricas é identificado por $\rho, \theta$ e $z$, onde

$$
\begin{gathered}
x=\rho \cos \theta \\
y=\rho \operatorname{sen} \theta \\
z=z
\end{gathered}
$$

Com isso, a circunferência do transdutor e a esfera centrada em $P$ passam a ser descritas, respectivamente, pelas Eqs. (6) e (7):

$$
\rho=a
$$

e

$$
\begin{gathered}
\left(\rho \cos \theta-\rho_{P} \cos \theta_{P}\right)^{2}+\left(\rho \operatorname{sen} \theta-\rho_{P} \operatorname{sen} \theta_{P}\right)^{2}+ \\
\left(z-z_{P}\right)^{2}=R^{2}
\end{gathered}
$$

onde $\rho_{P}, \theta_{P}$ e $z_{P}$ são as coordenadas cilíndricas do ponto $\mathrm{P}$.

A Fig. 5 ilustra as principais características das circunferências que se interseccionam no plano xy. Notase que a parte do arco pertencente à circunferência projetada, que se localiza no interior da circunferência do transdutor, contém as fontes que geram a mesma contribuição de pressão para o ponto P. Este arco pode ser calculado pelo produto do raio da circunferência projetada pelo dobro do ângulo formado por $r$ e $\rho_{p}$. Para fazer este cálculo basta utilizar a lei dos cossenos.

Aplicando-se a lei dos cossenos na disposição geométrica, mostrada na Fig. 5, obtém-se:

$$
a^{2}=r^{2}+\rho_{P}^{2}-2 r \rho_{P} \cos \alpha
$$

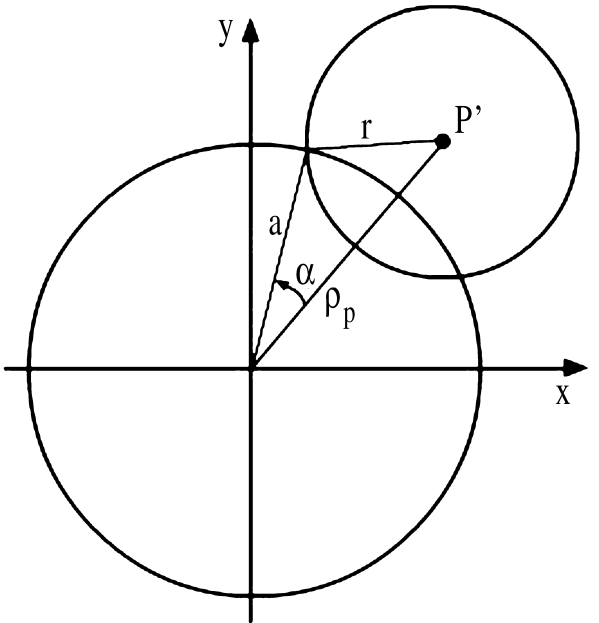

Figura 5 - Intersecção da circunferência do transdutor com a circunferência centrada em $\mathrm{P}^{\prime}$.

Portanto, a porção do arco que se encontra no interior da circunferência do transdutor é dada por:

$$
L=2 r \cdot \arccos \left(\frac{r^{2}+\rho_{P}^{2}-a^{2}}{2 r \rho_{P}}\right)
$$

Observando-se as Figs. 4 e 5, nota-se que, no caso (a) da Fig. 4, onde o ponto P' é externo, somente ocorrerá a intersecção apresentada na Fig. 5 quando $\rho_{P}-a<r<\rho_{P}+a$. O cálculo do arco resultante é feito pela Eq. (9).

No caso (b), apresentado na Fig. 4, a projeção de P encontra-se internamente a circunferência do transdutor. Há duas possibilidades neste caso:

b1) se $0<r<a-\rho$, então não há intersecção, mas a circunferência projetada encontra-se no interior do transdutor e, portanto, todo seu perímetro é constituído por fontes contribuintes de pressão em $\mathrm{P}, \operatorname{logo}$ $L=2 \pi r$

b2) se $\left|\rho_{P}-a\right| \leqslant r<\rho_{P}+a$, então ocorre a intersecção das circunferência e o arco é calculado por (9).

Em suma, deve-se fazer uma varredura de arcos sobre a superfície do transdutor, a qual demanda uma grande quantidade cálculos que, preferencialmente, devem ser executados através de computadores.

O campo acústico foi simulado por meios computacionais. Um programa foi desenvolvido junto ao software Matlab, versão 6.0 [24], baseado no seguinte algoritmo:

- definem-se as variáveis do problema, como características do transdutor e do meio de propagação; 
- definem-se os valores dos pontos nos quais deseja-se conhecer o campo acústico e os incrementos que devem separar esses pontos;

- gera-se o conjunto de pontos;

- para cada valor de pressão relacionado a um ponto do meio de propagação faz-se uma somatória dos elementos da intersecção entre a circunferência e a esfera;

- estabelece-se as condições de variação da variável $r$ de acordo com a posição do ponto em que se deseja conhecer o campo e os limites de valores assumidos por $r$.

- gera-se um gráfico bi e/ou tri-dimensional da pressão obtida em função dos pontos do campo.

\section{Resultados}

Nesta seção são apresentados gráficos tri-dimensionais das pressões acústicas em função de $y$ e $z$ resultantes de simulações do método utilizado. Em todos os casos considerou-se um transdutor com raio de $2,5 \mathrm{~cm}$, irradiando em um meio constituído de água, onde a velocidade de propagação é de $1500 \mathrm{~m} / \mathrm{s}$. Os resultados mostram os níveis de pressão em um semiplano formado pelos eixos $y$ (entre 0 e $1,5 a$ ) e $z$ (entre 0 e $a^{2} / \lambda$ ), onde $\lambda$ é o comprimento de onda definido por $\lambda=c / f$. Os transdutores são denominados T1, T2 e T3 e se caracterizam pela razão $a / \lambda$. A Tabela 1 apresenta os valores utilizados no software, apresentado no apêndice, para a obtenção dos resultados.

As Figs. 6, 7 e 8 mostram respectivamente os gráficos de T1, T2 e T3 obtidos com a simulação computacional.

Tabela 1 - Parâmetros empregados nas simulações dos campos acústicos.

\begin{tabular}{lccc}
\hline & $\mathrm{T} 1: a / \lambda=1,0$ & $\mathrm{~T} 2: a / \lambda=2,5$ & $\mathrm{~T} 3: a / \lambda=4,0$ \\
\hline Freqüência $[\mathrm{kHz}]$ & 60 & 150 & 240 \\
Divisões em z & 30 & 60 & 60 \\
Divisões em y & 30 & 60 & 60 \\
Incremento do raio r [\% de $a]$ & 0.01 & 0.01 & 0.01 \\
\hline
\end{tabular}

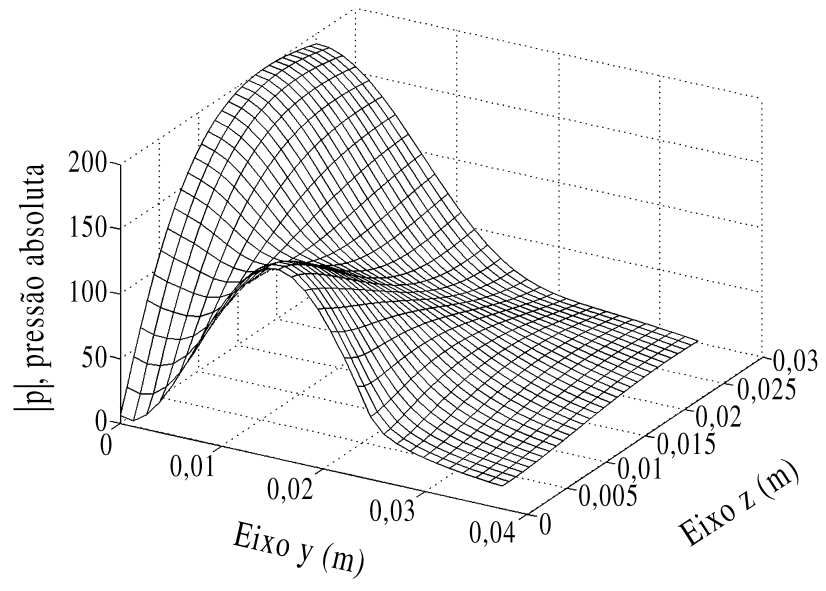

Figura 6 - Pressão acústica em função de y e z no campo próximo de um transdutor circular $(\mathrm{a} / \lambda)=1,0$.

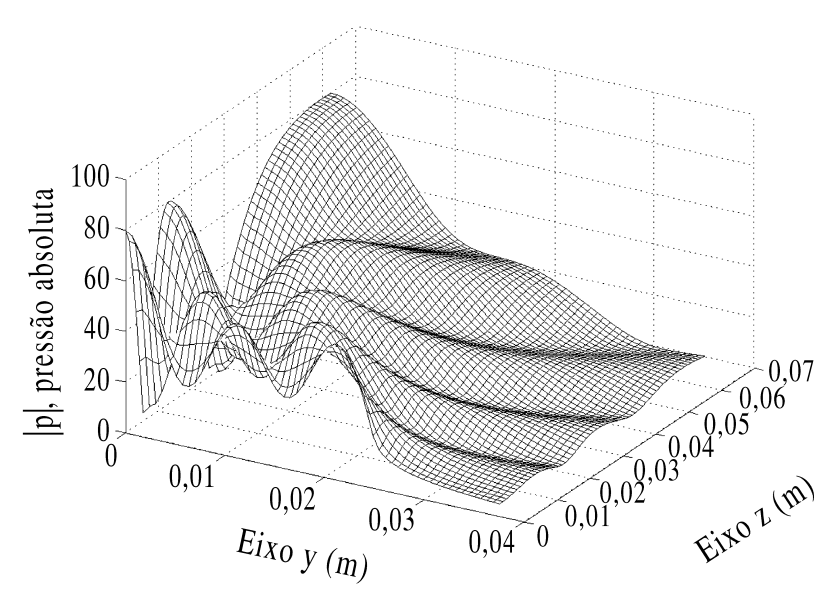

Figura 7 - Pressão acústica em função de y e z no campo próximo de um transdutor circular $(\mathrm{a} / \lambda)=2,5$. 


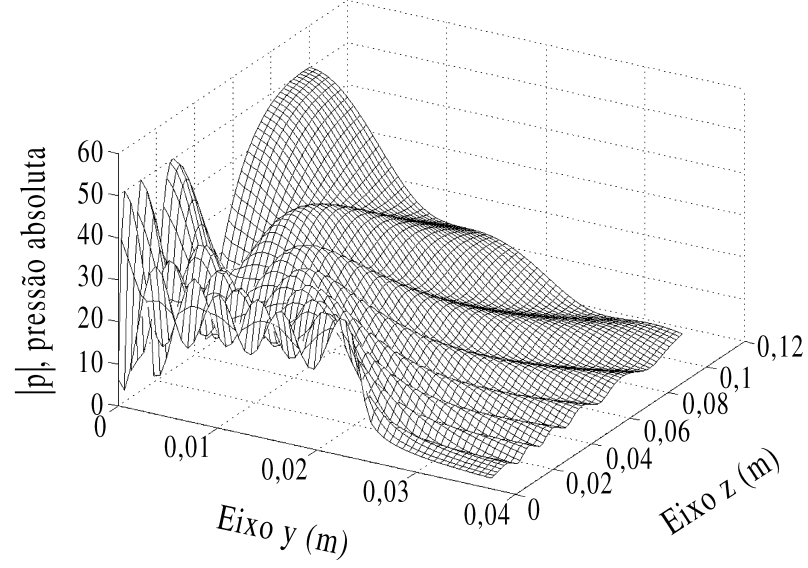

Figura 8 - Pressão acústica em função de y e z no campo próximo de um transdutor circular $(\mathrm{a} / \lambda)=4,0$.

O comportamento do campo acústico ao longo do eixo central perpendicular ao transdutor é apresentado nas Figs. 9, 10 e 11. Estas figuras são correspondentes, respectivamente, aos transdutores $\mathrm{T} 1, \mathrm{~T} 2 \mathrm{e} \mathrm{T} 3$.

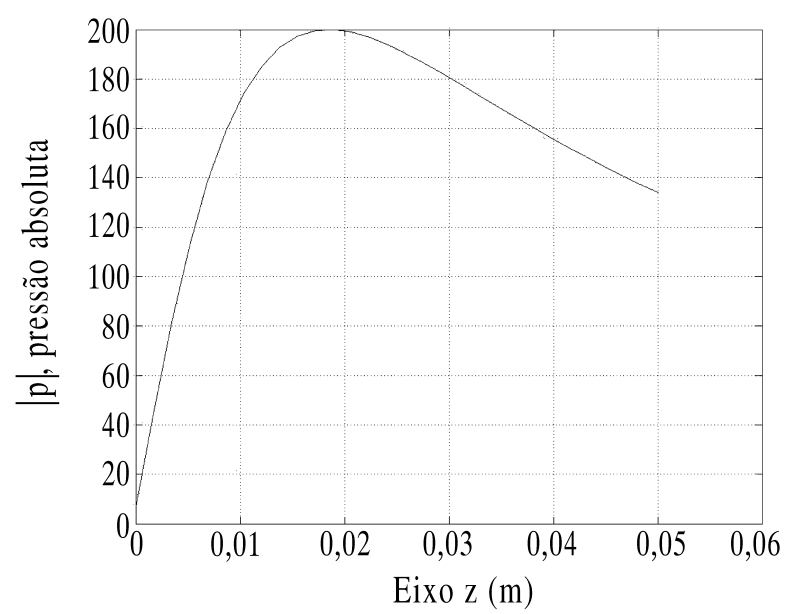

Figura 9 - Pressão acústica sob o eixo z no transdutor com a/ $\lambda=1,0$.

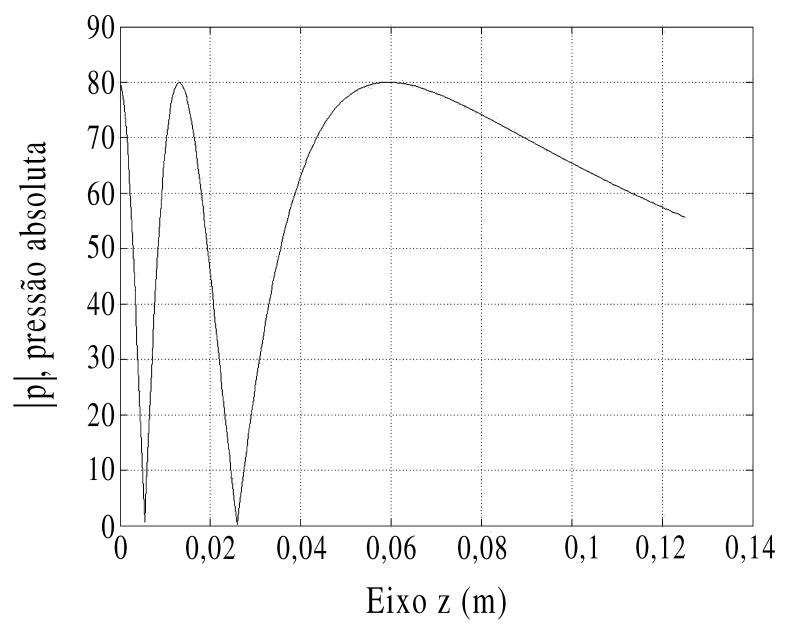

Figura 10 - Pressão acústica sob o eixo z no transdutor com $\mathrm{a} / \lambda=2,5$.

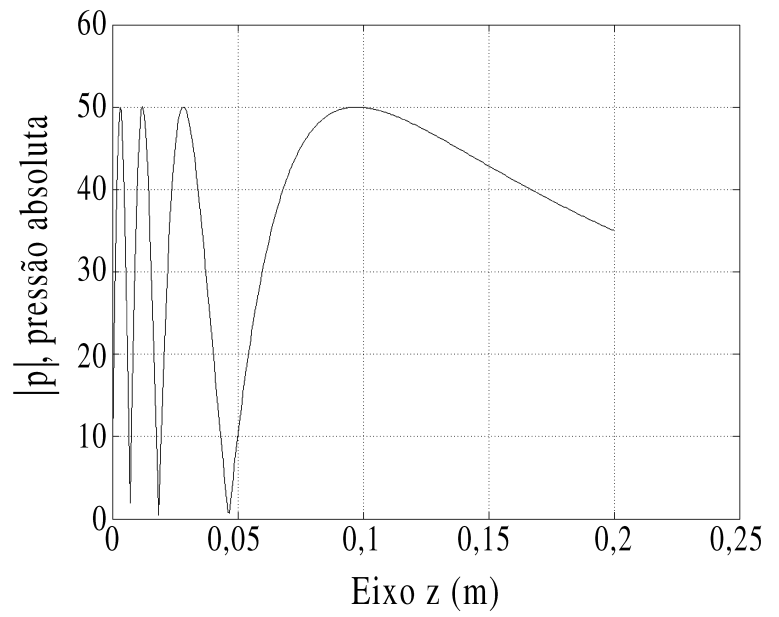

Figura 11 - Pressão acústica sob o eixo z no transdutor com $\mathrm{a} / \lambda=4,0$.

\section{Discussões e conclusões}

Foi apresentado um método para simulação computacional de campos acústicos. A temática está centrada em conceitos e na interpretação física da interferência de ondas que são amplamente discutidos em cursos introdutórios de Física. Procurou-se estabelecer um vínculo entre a modelagem de um problema de interferência com os fatores geométricos associados a ele e, nas simulações, de métodos numéricos e sua execução computacional. Os parâmetros de entrada das simulações podem ser variados de diferentes formas para que se possam explorar outras visões, mais específicas, da distribuição dos níveis de pressão do campo acústico.

Os gráficos apresentados nas Figs. 6, 7 e 8 mostram que nas proximidades da superfície do transdutor predomina uma acentuada alternância de valores mínimos e máximos, especialmente quando o valor da razão $a / \lambda$ é elevado. Afastando-se do transdutor pelo eixo central nota-se que, numa distância superior a aproximadamente $0,75 a / \lambda$, essa alternância deixa de existir e a pressão decai exponencialmente, tal como é notado nas Figs. 9, 10 e 11. Estas duas regiões são denominadas, respectivamente, campo próximo e campo distante. A alternância de valores mínimos e máximos é devida a diferença de fase das contribuições das fontes pontuais. Quando se afasta do transdutor os raios que ligam as fontes pontuais com o ponto $\mathrm{P}$ tornam-se praticamente paralelos e assim as contribuições passam a ter aproximadamente a mesma fase, logo os pontos com 
valores extremos deixam de existir. Em termos de ensino, isto pode ser estudado com maior profundidade estimulando-se uma discussão mais detalhada sobre este assunto, a qual pode ser realizada com auxílio de livros sobre óptica onde se abordam as difrações de Fresnel e Fraunhofer [25].

Os valores dos níveis de pressão foram determinados de modo relativo, uma vez que o valor da constante $\Psi$ não foi calculado. Este valor é dependente das características do meio de propagação e não influi na distribuição dos valores de pressão no campo, apenas afeta a amplitude obtida.

Outro aspecto bastante importante que pode ser explorado em atividades pedagógicas refere-se ao significado físico dos fasores utilizados para descrever a velocidade de vibração das fontes pontuais. Através dele pode-se enfatizar o uso de um ente matemático capaz de expressar uma grandeza em termos de amplitude e fase.

Em estudos mais específicos sobre a formação dos campos acústicos emprega-se a integral de Rayleigh [2]. Com a metodologia apresentada, a contribuição de um determinado conjunto de fontes infinitesimais e o elemento de arco surgido da resolução de um problema geométrico ficam vinculadas, o que permite associar com facilidade a interpretação física ao procedimento matemático.

Quanto ao trabalho matemático desenvolvido, deve-se enfatizar a praticidade da aplicação e interpretação das coordenadas cilíndricas. Todo o problema foi quantificado a partir de equações de circunferência, as quais, em coordenadas cilíndricas, simplificam substancialmente o trabalho algébrico. Muito embora este método de cálculo seja limitado a problemas de geometria circular, esse procedimento é vantajoso do ponto de vista do trabalho computacional, pois o programa necessita executar apenas uma somatória por ponto do meio de propagação, ao invés de duas, quando se aplicam os métodos diretamente ligados a integral de Rayleigh.

A parte computacional foi concebida através da modelagem do problema físico. Este aspecto é de suma importância, pois agrega o computador, o qual, por si só, é um grande atrativo aos alunos, como uma ferramenta de apoio ao ensino da Física e mostra as perspectivas e potencialidades do programa na resolução de problemas [26].

Empregaram-se várias instruções fundamentais típicas das linguagens de programação (ver Apêndice). Adotou-se o conhecido software Matlab, versão 6.0, todavia o uso de linguagens estruturadas pode ser adequado de acordo com o conteúdo desenvolvido nas disciplinas de programação de computadores, mas especialmente para estudantes de cursos da área de informática.

Os resultados também podem ser apresentados na forma de curvas de nível, através de instruções específicas do Matlab, isto contribui para introduzir este importante conceito matemático.

A quantidade de pontos utilizada e o incremento dado ao valor de $r$ devem ser aumentados quando a razão $a / \lambda$ aumentar. $\mathrm{O}$ campo próximo torna-se cada vez mais repleto de máximos e mínimos com o aumento de $a / \lambda$ logo, para conseguir melhores definições do campo, é necessário um cálculo mais preciso. Para ilustrar esta problemática aconselha-se reproduzir os resultados de T3 utilizando-se 30 divisões em cada eixo.

Do ponto de vista pedagógico, conclui-se que a atividade interdisciplinar proposta pode ser interessante no sentido de agrupar conhecimentos de 3 matérias fundamentais estudadas nos cursos introdutórios de ciências exatas e pode ser desenvolvida de forma conjunta ou separada pelos professores das matérias envolvidas. Esta proposta de trabalho já foi aplicada algumas vezes em cursos para engenheiros e tecnólogos e os resultados obtidos foram satisfatórios quanto ao desempenho e motivação dos alunos.

\section{Referências}

[1] V.B. Barbeta e I. Yamamoto, Rev. Bras. Ens. Fís. 24, 324 (2002).

[2] G. Kino, Acoustic Waves: Devices, Imaging and Ana$\log$ Signal Processing (Prentice Hall, New Jersey, 1987).

[3] G.R. Harris, J. Acoust. Soc. Am. 70, 10 (1980).

[4] P. Tipler, Física, (Livros Técnicos e Científicos, Rio de Janeiro, 2000), v. 1, $4^{a}$ edição.

[5] J. Zemanek, J. Acoust. Soc. Am. 49, part 2, 181 (1971).

[6] P. Stepanishen, J. Acoust. Soc. Am. 49 part 2, 1629 (1971).

[7] J.C. Lockwood and J.G. Willette, J. Acoust. Soc. Am. 53, 735 (1973).

[8] K.B. Ocheltree and L.A. Frizzell, IEEE Trans. Ultrason. Ferroelectr. Freq. Control 36, 242 (1989).

[9] L.X. Yao, J.A. Zabzebski nad E.J. Boote, IEEE Trans. Ultrason. Ferroelectr. Freq. Control 36, 446 (1989). 
[10] X. Fan, E. Moros and W. Straube, J. Acoust. Soc. Am. 102, 2734 (1997).

[11] J. D'hooge, J. Nuyts, B. Bijnens, B. De Man, P. Suetens, J. Thoen, M.C. Herregods and F. Van de Werf, J. Acoust. Soc. Am. 102, 78 (1997).

[12] C.T. Liauh and W.L. Lin, J. Acoust. Soc. Am. 105, 2243 (1999).

[13] J.A. Jensen, J. Acoust. Soc. Am. 105, 3266 (1999).

[14] P.R. Stepanishen and K.C. Benjamin, J. Acoust. Soc. Am. 71, 803 (1982).

[15] M.E. Schafer and P.A. Lewin, J. Acoust. Soc. Am. 85, 2202 (1989).

[16] Z.G. Hah and K.M. Sung, J. Acoust. Soc. Am. 92, 3403 (1992).

[17] C.J. Vecchio and P. Lewin, J. Acoust. Soc. Am. 95, 2399 (1994).

[18] P. Wu, R. Kazys and T. Stepinski, J. Acoust. Soc. Am. 99, 1339 (1996).

[19] P. Wu, R. Kazys and T. Stepinski, J. Acoust. Soc. Am. 99, 1349 (1996).

[20] P. Wu, R. Kazys and T. Stepinski, J. Acoust. Soc. Am. 101, 125 (1997).

[21] J.A. Gallego-Juárez, J. Phys. E. Sci. Instrum. 22, 804 (1989).

[22] F.J. Arnold, Mapeamento de Campos Ultra-Sônicos. Dissertação de Mestrado apresentada a FFCLRP-USP, 1990.

[23] R.J. Santos, Matrizes, Vetores e Geometria Analítica (Imprensa Universitária da UFMG, Belo Horizonte, 2001).

[24] S.J. Chapman, Programação em MATLAB para Eengenheiros (Thomson Learning, São Paulo, 2003).

[25] E. Hecht, Optics (Addison Wesley, New York, 1989).

[26] I. Yamamoto e V.B. Barbeta, Rev. Bras. Ens. Fís. 23, $215(2001)$

\section{Apêndice}

A seguir é apresentado um programa para simulação de campos acústicos desenvolvido no software Matlab versão 6.0 .

\%Programa para calculo de campos acusticos de transdutores circulares

$\%$ caracteristicas do transdutor e do meio de propagaçao

$\mathrm{a}=$ input('raio do transdutor $[\mathrm{m}]=. .$. '); f=input('frequencia de vibraçao do transdutor

$\left.[\mathrm{Hz}]=\ldots{ }^{\prime}\right)$;

c=input('velocidade de propagaçao de ondas do meio $[\mathrm{m} / \mathrm{s}]=\ldots$...);

lambda=c/f; \%comprimento de onda

$\%$ pontos do grafico

\%intervalo de $\mathrm{z}$

$\mathrm{zo}=$ input('valor minimo de $\mathrm{z}$ [unidades* $\left(\mathrm{a}^{\wedge}\right.$ 2)/lambda] $=\ldots$ (informar unidades)');

$\mathrm{zmin}=\mathrm{zo}^{*} \mathrm{a}^{\wedge}$ 2/lambda;

$\mathrm{zf}=$ input('valor maximo de $\mathrm{z}$ [unidades* $\left(\mathrm{a}^{\wedge}\right.$

2)/lambda] $=. .$.

(informar unidades)');

zmax $=z f^{*} a^{\wedge}$ 2/lambda;

\%intervalo de y

yo=input('valor minimo de y [unidades*a]=...(informar unidades)');

ymin=yo*a;

yf=input('valor maximo de y [unidades*a]=...(informar unidades)');

ymax $=y f * a ;$

$\%$ divisoes

jmax =input('numero de divisoes de $\mathrm{z}=\ldots$...');

kmax=input('numero de divisoes de $\mathrm{y}=. .$. ');

\%incremento do raio

dr=input('comprimento do incremento do raio

[valor*a]=...');

$\mathrm{dr}=\mathrm{dr} * \mathrm{a}$

\%loop dos pontos

for $\mathrm{j}=1: \mathrm{jmax}$

zmin=zmin+eps;

$\%$ eps e um incremento minimo para evitar indeterminaçoes

$\mathrm{Az}=(\mathrm{zmax}-\mathrm{zmin}) /(\mathrm{jmax}-1)$;

$\mathrm{z}(\mathrm{j})=A z^{*}(\mathrm{j}-1)+\mathrm{zmin} ;$

for $\mathrm{k}=1: \mathrm{kmax}$

$\mathrm{Ay}=(\mathrm{ymax}-\mathrm{ymin}) /(\mathrm{kmax}-1)$;

$\mathrm{y}(\mathrm{k})=\mathrm{Ay} *(\mathrm{k}-1)+\mathrm{ymin}$

\%valor inicial da pressao no ponto $\mathrm{y}(\mathrm{k}), \mathrm{z}(\mathrm{j})$

$\mathrm{p}(\mathrm{j}, \mathrm{k})=0$;

\%estudo de casos

if $\operatorname{abs}(\mathrm{y}(\mathrm{k}))<\mathrm{a} \%$ ponto interno ao transdutor

$\mathrm{r}=\mathrm{dr}$;

while $\mathrm{r}<=(\mathrm{a}+\mathrm{abs}(\mathrm{y}(\mathrm{k})))$

if $\mathrm{r}<=(\mathrm{a}-\mathrm{abs}(\mathrm{y}(\mathrm{k})))$

$\mathrm{L}=2 * \mathrm{pi} * \mathrm{r}$

else

$\mathrm{L}=\mathrm{r}^{*} 2 * \operatorname{acos}\left(\left((\mathrm{r} 2)+\left(\mathrm{y}(\mathrm{k})^{\wedge} 2\right)-\left(\mathrm{a}^{\wedge} 2\right)\right) /\left(2 * \mathrm{r}^{*} \mathrm{y}(\mathrm{k})\right)\right) ;$

end

$\%$ Calculo da pressao (somatoria) 
$\mathrm{R}=\operatorname{sqrt}\left(\mathrm{r}^{\wedge} 2+\mathrm{z}(\mathrm{j})^{\wedge} 2\right)$ $\mathrm{v}=\exp (\mathrm{i} * 2 * \mathrm{pi} * \mathrm{f} * \mathrm{R} / \mathrm{c})$;

$\mathrm{p}(\mathrm{j}, \mathrm{k})=\mathrm{p}(\mathrm{j}, \mathrm{k})+\left(\mathrm{L}^{*} \mathrm{v} / \mathrm{R}\right)$;

$\mathrm{r}=\mathrm{r}+\mathrm{dr}$;

end

else \%ponto externo ao transdutor

$\mathrm{r}=\mathrm{dr}+\mathrm{abs}(\mathrm{y}(\mathrm{k})-\mathrm{a})$;

while $\mathrm{r}<=(\mathrm{y}(\mathrm{k})+\mathrm{a})$

$\mathrm{L}=\mathrm{r}^{*} 2 * \operatorname{acos}\left(\left(\left(\mathrm{r}^{\wedge} 2\right)+\left(\mathrm{y}(\mathrm{k})^{\wedge} 2\right)-\left(\mathrm{a}^{\wedge} 2\right)\right) /\left(2^{*} \mathrm{r}^{*} \mathrm{y}(\mathrm{k})\right)\right)$;

$\%$ Calculo da pressao (somatoria)

$\mathrm{R}=\operatorname{sqrt}\left(\mathrm{r}^{\wedge} 2+\mathrm{z}(\mathrm{j})^{\wedge} 2\right)$

$\mathrm{v}=\exp (\mathrm{i} * 2 * \mathrm{pi} * \mathrm{f} * \mathrm{R} / \mathrm{c})$;

$\mathrm{p}(\mathrm{j}, \mathrm{k})=\mathrm{p}(\mathrm{j}, \mathrm{k})+(\mathrm{L} * \mathrm{v} / \mathrm{R})$;

$\mathrm{r}=\mathrm{r}+\mathrm{dr}$;

end

end

end end

\%grafico da pressao acustica em funçao de y e z (grafico tridimensional)

$\mathrm{h}=\operatorname{mesh}(\mathrm{y}, \mathrm{z}, \mathrm{abs}(\mathrm{p}))$;

ylabel('eixo z (m)'), xlabel('eixo y (m)'), zlabel(' $|\mathrm{p}|$, pressao absoluta'), view $(30,35)$;

set(h,'EdgeColor','k','FaceColor','w')

set(gcf,'Color','w')

set(gca,'XColor','k','YColor,',k','ZColor','k')

$\%$ grafico da pressao acustica sobre o eixo central z (grafico bidimensional)

figure(2);

$\mathrm{pp}=\mathrm{abs}(\mathrm{p})$;

\%para $\mathrm{y}=0$, corresponde $\mathrm{y}(1)$

$\mathrm{pz}=\mathrm{pp}(:, 1)$;

plot(z,pz); grid; xlabel('eixo z(m)'), ylabel('|p|, pressao absoluta'); 